\title{
Explicit Expressions of Impedances and Wake Functions
}

\author{
K.Y. Ng \\ Fermilab, Batavia, IL 60510 \\ K.Bane \\ SLAC, Stanford, CA 94309
}

(October, 2010)

\begin{abstract}
Sections 3.2.4 and 3.2.5 of the Handbook of Accelerator Physics and Engineering on Landau damping are combined and updated. The new addition includes impedances and wakes for multi-layer beam pipe, optical model, diffraction model, and cross-sectional transition.
\end{abstract}

Submitted to

3rd edition of Handbook of

Accelerator Physics and Engineering 


\subsubsection{Explicit Expressions of Impe- dances and Wake Functions K.Y. Ng, FNAL, K. Bane, SLAC}

See tables in the next pages.

\section{References}

[1] A.W. Chao, Physics of Collective Beam Instabilities in High Energy Accelerators, Wiley (1993) ch.2

[2] K.Y. Ng, Fermilab-FN-0756, 2004

[3] K. Bane, M. Sands, Proc. Micro Bunches Workshop 1995, p. 131

[4] S. Krinsky et al, PRST-AB 7 (2004) 114401 Proc. Micro Bunches Workshop 1995, p. 131

[5] A. Piwiniski, DESY 94-068 (1994); DESY 84097 (1984)

[6] A. Piwiniski, PAC 77, p.1364, X.E. Lin, SLACPUB-7924 (1998)

[7] L. Palumbo and V.G. Vaccaro, Nuovo Cimento A 89, 3 (1985)

[8] R. Gluckstern, et al, Phys Rev E 47,656 (1993)

[9] K. Yokoya, PA 41, 221 (1993)

[10] A. Lutman, et al, PRST-AB 11, 074401 (2008).

[11] K.Y. Ng, PA 16, 63 (1984)

[12] N. Mounet and E. Métral, CERN-BE2009-039 (2009). For a Mathematica code to compute multi-layer impedances, visit https://impedance.web.cern.ch/impedance /Codes/ReWall/Rewall_to_date.zip and https://impedance.web.cern.ch/impedance Note that we separate impedances into the usual space-charge (self-field plus wall image, both $\rightarrow 0$ as $\gamma \rightarrow \infty$ ) and wall impedances, whereas Mounet separates them instead into self-direct (due to beam only but excluding wall image) and wall (our usual wall impedance plus wall image).

[13] B. Zotter, CERN-AB-2005-043 (20050

[14] A. Burov and V. Lebedev, EPAC 02 p.1402

[15] G. Stupakov et al, PRST-AB 10 (2007) 054401; K. Bane et al, PRST-AB 10 (2007) 074401

[16] S.A. Heifets, PRD 40 (1989) 3097; S.A. Heifets, S.A. Kheifets, Rev. Mod. Phys. 63 (1990) 631

[17] E. Gianfelice, L. Palumbo, IEEE Tr. NS 37, 2 (1990) 1081

[18] I. Zagorodnov, K. Bane, Proc. EPAC 06, p. 2859

[19] J. Lawson, Rutherford Report RHEL/M 144 (1968); K. Bane, M. Sands, SLAC-PUB-4441 (1987)
[20] R. Gluckstern, PRD 39 (1989) 2773, 2780

[21] G. Stupakov, PAC95, p.3303

[22] K. Yokoya and K. Bane, PAC 99, p.1725

[23] A. Fedetov, R. Gluckstern, M. Venturini, PRST-AB 2 (1999) 064401

[24] K. Bane et al, Proc. ICAP 98, p.137

[25] K. Bane, SLAC-PUB (2003) 9663

[26] M. Sands, SLAC note PEP-253 (1977); H.A. Bethe, PR 66 (1944) 163

[27] S.S. Kurennoy, PRE 55 (1997) 3529; S.S. Kurennoy, R.L. Gluckstern, ibid (1997) 3533

[28] S.S. Kurennoy, PA 39 (1992) 1; PA 50 (1995) 167; R.L. Gluckstern, PRA 46, 1106, 1110 (1992); S.S. Kurennoy, R.L. Gluckstern, G.V. Stupakov, PRE 52 (1995) 4354

[29] A. Fedotov, PhD Thesis, U. Maryland (1997)

[30] S.S. Kurennoy, G.V. Stupakov, PA 45 (1994) 95

[31] A. Novokhatski, A. Mosnier, PAC 97, p.1661

[32] K. Bane, A. Novokhatski, SLAC-AP-117 (1999)

[33] G. Stupakov, Proc. 19th Advanced ICFA Beam Dynamics Workshop (Arcidosso, 2000), p.141

[34] K.Y. Ng, PRD 42 (1990) 1819; A. Burov and A. Novokhatski, HEAC 92, p.537

[35] G.V. Stupakov, PRST-AB 1 (1998) 064401

[36] K. Bane, et al, PAC 97, p 1738

[37] J.B. Murphy et al, PAC 95, p.2980; PA 57 (1997) 9

[38] Y. Derbenev et al, DESY-TESLA-FEL 95-05 (1995)

[39] R. Warnock, P. Morton, SLAC-PUB-4562 (1988); R. Warnock, Proc. 4th Advanced ICFA Beam Dynamics Workshop (1990) p.151

[40] K. Bane, P. Morton, LINAC 86, p.490

[41] K. Yokoya, CERN-SL-90-88-AP (1988)

[42] G.V. Stupakov, SLAC-PUB-7167 (1996)

[43] E. Keil, B. Zotter, PA 3 (1972) 11; K.Y. Ng, Fermilab Report FN-389 (1981)

[44] T. Weiland and B. Zotter, PA 11 (1981) 143

[45] G. Dome, PAC 85, p.2531

[46] S.A. Heifets and S.A. Kheifets, PA 25 (1990) 61; A. Hofmann, T. Risselada, and B. Zotter, Proc. 4th ICFA Beam Dynamics workshop (1990) p.138

[47] K.Y. Ng, R. Warnock, PAC 89, p.798; PRD 40 (1989) 231

[48] K.Y. Ng, PA 23 (1988) 93

[49] T. Toyama, et al, HB2006, p.140; G. Nassibian, CERN/PS 84-25 (BR) (1984); CERN 85-68 (BR) (1986)

[50] A. Piwinski, DESY Report 72/72 (1972) 


\section{General Remarks and Notation:}

In cylindrically symmetric structures $W_{m}^{\prime}(z)$ and $W_{m}(z)$ denote, respectively, $m$-th azimuthal multipole longitudinal and transverse wake functions, generated by point charge $Q$, at distance $-z>0$ behind. $W_{m}^{\prime}(z) \equiv d W_{m}(z) / d z$. $W_{m}^{\prime}(z)=0$ and $W_{m}(z)=0$ when $z>0$ when particle travels at the speed of light. $W_{m}^{\prime}(0)=\frac{1}{2} \lim _{z \rightarrow 0^{-}} W_{m}^{\prime}(z)$. Longitudinal and transverse momentum kicks on test charge $q$ near pipe axis: $\Delta p_{\|}(z)=-q Q W_{0}^{\prime}(z) / c$, $\Delta p_{\perp}(z)=-q Q \tilde{r}_{\perp} W_{1}(z) / c$, where $\tilde{r}_{\perp}$ is (small) offset of the source or exciting charge.

The $m$-th multipole longitudinal impedance $Z_{m}^{\|}(k)=\int e^{-i k z / \beta} W_{m}^{\|}(z) d z /(\beta c)$ is related to the $m$-th multipole transverse impedance, $Z_{m}^{\perp}(k)=i \int e^{-i k z / \beta} W_{m}^{\perp}(z) d z /\left(\beta^{2} c\right)$, by $Z_{m}^{\|}=k Z_{m}^{\perp}$ $(m \neq 0)$, where $k=\omega / c$. Note that $Z_{m}^{\|}(-k)=Z_{m}^{\| *}(k), Z_{m}^{\perp}(-k)=-Z_{m}^{\perp *}(k)$.

For periodic or translationally invariant structures: steady-state results are given per length $L$. Unless otherwise stated, structures are cylindrically symmetric with perfectly conducting metallic walls, and with beam pipes of radius $b$. In many cases, $\beta=v / c$ has been set to 1 . $Z_{0}=\sqrt{\mu_{0} / \epsilon_{0}} \approx 377 \Omega$ is impedance, $\epsilon_{0}$ electric permittivity, and $\mu_{0}$ magnetic permeability of free space. 'Pill-box cavity' signifies a pill-box with beam pipes. Here $[\alpha \pm i|\beta|]^{1 / n}$ (with $\alpha, \beta$ real, $n=2$ or 3 ) is in the 1st/4th quadrant. $H(x)=0,1$ for $x \lessgtr 0$.

For 3D structures with mirror symmetry in $x$ and $y$, near axis momentum kick in $y$, $\Delta p_{y}=-q Q\left(\tilde{y} W_{d}^{y}+y W_{q}^{y}\right)$, with $\tilde{y}(y)$ offset of exciting (test) charge, and $W_{d}^{y}\left(W_{q}^{y}\right)$ dipole (quad) wake terms. Total $y$ wake $W_{y}=W_{d}^{y}+W_{q}^{y}$; total $y$ impedance $Z_{y}=Z_{d}^{y}+Z_{q}^{y}$.

\begin{tabular}{|c|c|c|c|}
\hline \multirow[b]{2}{*}{$\begin{array}{l}\text { Description } \\
\text { Space-charge: }[1] \\
\text { beam radius } a \text { in a } \\
\text { perfectly conducting } \\
\text { beam pipe of radius } \\
b, \text { transverse distri- } \\
\text { bution uniform. } \\
\end{array}$} & \multicolumn{2}{|l|}{ Impedances } & wake \\
\hline & $\begin{array}{l}\frac{Z_{0}^{\|}}{L}=i \frac{Z_{0} k g_{0}}{4 \pi \beta^{2} \gamma^{2}} \quad g_{0}=1 \\
\frac{Z_{m \neq 0}^{\perp}}{L}=i \frac{Z_{0}}{2 \pi \beta^{2} \gamma^{2} m}\left[\frac{1}{a^{2 m}}\right.\end{array}$ & $\begin{array}{l}2 \ln \frac{b}{a} \\
\left.-\frac{1}{b^{2 m}}\right]\end{array}$ & $\begin{array}{l}=\frac{Z_{0} c}{4 \pi \gamma^{2}}\left[1+2 \ln \frac{b}{a}\right] \delta^{\prime}(z) \\
=\frac{Z_{0} c}{2 \pi \gamma^{2} m}\left[\frac{1}{a^{2 m}}-\frac{1}{b^{2 m}}\right] \delta(z)\end{array}$ \\
\hline $\begin{array}{l}\text { Nonuniform distri- } \\
\text { butions: }[2] \quad a_{\text {eff }}^{\|} \text {is } \\
\text { equivalent-uniform- } \\
\text { beam radius, } g_{0}= \\
1+2 \ln \left(b / a_{\text {eff }}^{\|}, \text {while }\right. \\
a_{\text {eff }}^{\perp}[\pi \lambda(0)]^{-1 / 2} \text { is } \\
\text { the same when self- } \\
\text { force part written as } \\
1 / a^{\perp 2} \gamma_{e} \approx 0.57721\end{array}$ & $\begin{array}{l}\text { Distribution } \lambda(r) \\
\frac{3}{2 \pi \hat{r}}\left(1-\frac{r^{2}}{\hat{r}^{2}}\right)^{1 / 2} H(\hat{r}-r) \\
\frac{1}{2 \pi \hat{r}^{2}}\left(1-\frac{r^{2}}{\hat{r}^{2}}\right) H(\hat{r}-r) \\
\frac{2 \pi}{\pi^{2}-4} \cos ^{2} \frac{\pi r}{2 \hat{r}} H(\hat{r}-r) \\
\frac{1}{2 \pi \sigma_{r}^{2}} e^{-r^{2} /\left(2 \sigma_{r}^{2}\right)}\end{array}$ & $\begin{array}{l}1.921+2 \ln \frac{b}{\hat{r}} \\
\gamma_{e}+2 \ln \frac{b}{\sqrt{2} \sigma_{r}}\end{array}$ & $\frac{a_{\mathrm{eff}}^{\|}(m=0) \quad a_{\mathrm{eff}}^{\perp}(n}{0.8692 \hat{r}} \sqrt{\frac{2}{2}}$ \\
\hline
\end{tabular}

Image part of $Z_{1}^{\perp}$ can be written in terms of Laslett's electric image coefficients as $1 / b^{2} \rightarrow 2\left(\xi_{1 x, y}-\epsilon_{1 x, y}\right) / h^{2}$ with $h$ denoting half height of vacuum chamber. See Sec.??.

Resistive wall: [1, 3] wall thickness $t$, $\mathrm{dc}$ and ac conductivities $\sigma_{c}, \tilde{\sigma}_{c}$, relaxation time $\tau$; assume $|k| b \gg\left(s_{0} / b\right)^{3}$, thick walls: $t \gg \delta_{c}=$ $\sqrt{2 /\left(|k| Z_{0} \mu_{r} \sigma_{c}\right)}$, the skin depth.

$$
\begin{aligned}
& \frac{Z_{m}^{\|}}{L}=\frac{Z_{0} /\left(\pi b^{2 m+1}\right)}{\left(1+\delta_{m 0}\right) \sqrt{\frac{i Z_{0} \tilde{\sigma}_{c}}{k \mu_{r}}}-\frac{i b k}{m+1}}, \mu_{r}=\left\{\begin{array}{l}
\text { relative magnetic } \\
\text { permeability }
\end{array}\right. \\
& \tilde{\sigma}_{c}=\sigma_{c} /(1-i k c \tau)
\end{aligned}
$$

Typically, $\tau=27 / 40 / 8$ fs for $\mathrm{Cu} / \mathrm{Ag} / \mathrm{Al}$. Valid for $c \tau / s_{0} \ll 1$, characteristic distance $s_{0}=\left[2 b^{2} \mu_{r} /\left(Z_{0} \sigma_{c}\right)\right]^{1 / 3}, \alpha=\left[(m+1)\left(1+\delta_{m 0}\right) / 2\right]^{2 / 3}$.

$$
\frac{W_{m}^{\prime}}{L}=\frac{4 Z_{0} c(m+1)}{\pi b^{2 m+2}}\left[\frac{e^{\alpha z / s_{0}}}{3} \cos \left(\frac{\sqrt{3} \alpha z}{s_{0}}\right)-\frac{\sqrt{2}}{\pi} \int_{0}^{\infty} d x \frac{x^{2} e^{\alpha z x^{2} / s_{0}}}{x^{6}+8}\right]
$$




\begin{tabular}{|c|c|}
\hline Description & Impedances \\
\hline $\begin{array}{l}\text { Low frequency: }[1] \\
k \ll 1 / s_{0} \text {, long range } \\
|z| \gg s_{0}\end{array}$ & \begin{tabular}{c|c}
$\frac{Z_{m}^{\|}}{L}=\frac{1-\operatorname{sgn}(k) i}{\left(1+\delta_{0 m}\right) \pi \sigma_{c} \delta_{c} b^{2 m+1}}$ & $\frac{W_{m}^{\prime}}{L}=\frac{-c}{2 \pi b^{2 m+1}\left(1+\delta_{m 0}\right)} \sqrt{\frac{Z_{0} \mu_{r}}{\pi \sigma_{c}}} \frac{1}{(-z)^{3 / 2}}$ \\
Note $: \quad Z_{1}^{\perp}=\frac{2}{b^{2} k} Z_{0}^{\|}$, & $W_{1}=\frac{2}{b^{2}} \int W_{0}^{\prime} d z$
\end{tabular} \\
\hline $\begin{array}{l}\text { Low freque } \\
\text { wall: }[1] t< \\
|k| \ll 1 / \sqrt{b t}\end{array}$ & $\frac{Z_{0}^{\|}}{L}=-\frac{Z_{0} k t}{2 \pi b}, \quad \frac{Z_{1}^{\perp}}{L}=-i \frac{Z_{0} t}{\pi b^{3}} \quad \frac{W_{0}^{\prime}}{L}=-\frac{Z_{0} t c}{2 \pi b} \delta^{\prime}(z), \frac{W_{1}}{L}=-\frac{Z_{0} c t}{\pi b^{3}} \delta(z)$ \\
\hline $\begin{array}{l}\text { High frequency: }[3] \\
k \gtrsim 1 / s_{0}, \text { short range } \\
|z| \lesssim s_{0}, \text { with } c \tau \gtrsim s_{0} . \\
k_{n}=\sqrt{Z_{0} \sigma_{c} / c \tau} \text { is }\end{array}$ & $\begin{array}{l}\frac{Z_{m}^{\|}}{L}=\frac{4 Z_{0} c \tau(m+1)}{\pi b^{2 m+1}} \\
\quad \times \frac{1-4 i k c \tau}{b(1-4 i k c \tau)^{2}+32 k_{p}(\alpha c \tau)^{2}}\end{array}$ \\
\hline plasma frequency $/ c$. & $\begin{array}{l}\text { These formulae depend only on the plasma frequency of } \\
\text { Effects of relative magnetic permeability have not been }\end{array}$ \\
\hline $\begin{array}{l}\text { Finite length, lossy } \\
\text { insert: [4] of length } \\
L, \text { in lossless pipe }\end{array}$ & If $b^{2} / s_{0}^{3} k^{2} \ll L \ll k b^{2}, \quad Z_{0}^{\|}=\frac{2^{1 / 2} Z_{0}}{\pi^{3 / 2} b} \sqrt{\frac{i L}{k}}, \quad$ else $Z_{0}^{\|}$as given above \\
\hline $\begin{array}{l}\text { Displaced beam: }[5] \\
\text { at } \vec{a}=\left(a_{x}, a_{y}\right) \text {, rms } \\
\text { bunch length } \sigma_{\ell} \text {, aver- } \\
\text { age current } I_{b}, \text { and } \\
\left(b / k^{2}, b, b-a\right) \gg \delta_{c} \\
\text { and } \gamma \gg 1\end{array}$ & $\begin{array}{l}\text { Wall impedances in last section multiplied by } f_{z} \text { for } Z_{0}^{\|} \text {and } f_{x, y} \text { for } \\
Z_{1}^{x, y} \text { with } f_{z}=\frac{b^{2}+a^{2}}{b^{2}-a^{2}}, \quad f_{x}=\frac{b\left(b^{2}-a^{2}+4 a_{x}^{2}\right)}{\left(b^{2}-a^{2}\right)^{3}}, \quad f_{y}=\frac{b\left(b^{2}-a^{2}+4 a_{y}^{2}\right)}{\left(b^{2}-a^{2}\right)^{3}} \\
\text { Power loss per length traversed is } \frac{P}{L}=\frac{\Gamma\left(\frac{3}{4}\right) I_{b}^{2}}{4 \pi^{2} b \sigma_{\ell}^{3 / 2} \sqrt{2 \mu_{r} \sigma_{c} / Z_{0}}} f_{z}\end{array}$ \\
\hline $\begin{array}{l}\text { Displaced beam } \\
\text { between two infi- } \\
\text { nite plates: }[5] \text { at } \\
y= \pm h / 2 . \gamma \gg 1 \\
{\left[h / k^{2}, h-2 y_{0}\right] \gg \delta_{c} \text {. }} \\
\text { Thin dielectric coat- } \\
\text { ing of thickness } \Delta h \text {. }\end{array}$ & $\begin{array}{l}Z_{0}^{\|}=\frac{1-\operatorname{sgn}(\omega) i}{\pi h} \sqrt{\frac{|\omega| \mu_{r} Z_{0}}{2 c \sigma_{c}}} f_{z}, \quad Z_{1}^{\perp}=\frac{\pi(\operatorname{sgn}(\omega) 1-i)}{\sqrt{2|\omega| \sigma_{c} /\left(c \mu_{r} Z_{0}\right)}} f_{\perp} \\
f_{z}=1+\frac{\pi y_{0}}{h} \tan \frac{\pi y_{0}}{h}, \quad f_{\perp}=\frac{f_{z}}{h^{3} \cos ^{2}\left(\pi y_{0} / h\right)}, \quad \text { beam at } y=y_{0} \\
Z_{0}^{\|}=-\frac{i \omega Z_{0}\left(\epsilon_{r} \mu_{r}-1\right) \Delta h}{\pi c \epsilon_{r} h} f_{z}, \quad Z_{1}^{\perp}=-\frac{i \pi Z_{0}\left(\epsilon_{r} \mu_{r}-1\right) \Delta h}{\epsilon_{r}} f_{\perp}\end{array}$ \\
\hline $\begin{array}{l}\text { Metallic coating } \\
\text { on ceramic pipe: }[6] \\
\text { compared with all } \\
\text { metal pipe } Z_{0}^{\|} \text {(met). } \\
t_{m, c}=\text { metal/ceramic } \\
\text { thickness } \ll b . \gamma \gg 1, \\
{\left[\left(\epsilon_{r}-1\right) t_{c}^{2},\left(1-\epsilon_{r}^{-1}\right) b t_{c}\right]} \\
\ll \sigma_{\ell}^{2} \text {. Loss } P / L \text { is } \\
\max \text { at } V=0.82 \text {. }\end{array}$ & $\begin{array}{l}Z_{0}^{\|}=Z_{0}^{\|}(\text {met }) \frac{A+\tanh \left(\nu t_{m}\right)}{1+A \tanh \left(\nu t_{m}\right)}, \quad A=\left(1-\frac{1}{\epsilon_{r}}\right) \nu t_{c}, \quad \nu=\frac{1-\operatorname{sgn}(\omega) i}{\delta_{c}} \\
\frac{P}{L}=\frac{Z_{0} I_{b}^{2} t_{c}\left(\epsilon_{r}-1\right)}{4 \sqrt{\pi} b \sigma_{\ell}^{2} \epsilon_{r}}\left[V-\sqrt{\pi} V^{2} e^{V^{2}} \operatorname{erfc}(V)\right], \quad V=\frac{\epsilon_{r} \sigma_{\ell}}{\left(\epsilon_{r}-1\right) Z_{0} \sigma_{c} t_{m} t_{c}} \\
\text { Field penetration through pipe, } \frac{E_{z, \text { out }}}{E_{z, \text { in }}}=\frac{1}{\sqrt{1+4\left(1-1 / \epsilon_{r}\right) t_{m} t_{c} / \delta_{c}^{2}}}, \\
\text { becomes significant when } t_{m} \lesssim t_{\text {crit }}=\delta_{c}^{2} / t_{c} . P / L \text { is at maximum at } \\
t_{\text {crit. }}\end{array}$ \\
\hline $\begin{array}{l}\text { Elliptical bea } \\
\text { pipe: }\end{array}$ & Low frequency, see $[7,8,5]$, high frequency, see $[9,10]$. \\
\hline $\begin{array}{l}\text { Recta } \\
\text { beam }\end{array}$ & Low frequency, see $[8]$, high frequency, see $[9,11]$. \\
\hline
\end{tabular}


Multi-layer pipe wall impedances: $[12,13]$ Cylindrical beam pipe with $N$ layers, $p$ th layer between $b^{(p-1)}<r<b^{(p)}$ and $b^{(N)} \rightarrow \infty$. Layer 1 is vacuum, $a<r<b^{(1)}$, with particle beam of charge $Q$ at $r=a$ and $\theta=0 . r<a$ is called the 0-th layer. Each layer has its own wavenumber $\nu=k \sqrt{1-\beta^{2} \varepsilon_{1} \mu_{1}}, k=\omega / v$ and own properties $\epsilon=\epsilon_{0} \varepsilon_{1}=\epsilon_{0} \epsilon_{r}\left(1+i \tan \vartheta_{\mathrm{E}}\right)-\frac{\sigma_{\mathrm{dc}}}{i \omega(1-i \omega \tau)}$, $\mu=\mu_{0} \mu_{1}=\mu_{0} \mu_{r}\left(1+i \tan \vartheta_{\mathrm{M}}\right) ; \vartheta_{\mathrm{E}}, \vartheta_{\mathrm{M}}$ are loss angles, $\sigma_{\mathrm{dc}}$ dc conductivity, and $\tau$ relaxation time. Actually any frequency dependent $\epsilon, \mu$, and conductivity can be assumed. Inside vacuum, $\nu=k / \gamma$; inside conducting metal of skin depth $\delta_{c}, \nu \approx(1-i) / \delta_{c}$. A user-friendly Mathematica code for computation is available [12]. The derivation is outlined briefly below. In terms of Bessel and Kelvin functions, $m$ th multipole longitudinal fields inside $p$-th layer: $E_{s}^{(p)}=\cos m \theta e^{i k s}\left[C_{I e}^{(p)} I_{m}\left(\nu^{(p)} r\right)+C_{K e}^{(p)} K_{m}\left(\nu^{(p)} r\right)\right], \quad \vec{E}$ is electric field $G_{s}^{(p)}=\sin m \theta e^{i k s}\left[C_{I g}^{(p)} I_{m}\left(\nu^{(p)} r\right)+C_{K g}^{(p)} K_{m}\left(\nu^{(p)} r\right)\right], \quad \vec{G}=Z_{0} \vec{H}, \vec{H}$ is magnetic field Matching $E_{s}, E_{\theta}, G_{s}$, and $G_{\theta}$ at boundary $r=b^{(p)}$ between $p$-th and $(p+1)$-th layers gives $\left[\begin{array}{l}C_{I e}^{(p+1)} \\ C_{K e}^{(p+1)} \\ C_{I g}^{(p+1)} \\ C_{K g}^{(p+1)}\end{array}\right]=M_{p}^{p+1}\left[\begin{array}{l}C_{I e}^{(p)} \\ C_{K e}^{(p)} \\ C_{I g}^{(p)} \\ C_{K g}^{(p)}\end{array}\right] \stackrel{\text { iteratively }}{\Longrightarrow}\left[\begin{array}{l}C_{I e}^{(N)} \\ C_{K e}^{(N)} \\ C_{I g}^{(N)} \\ C_{K g}^{(N)}\end{array}\right]=\mathcal{M}\left[\begin{array}{l}C_{I e}^{(1)} \\ C_{K e}^{(1)} \\ C_{I g}^{(1)} \\ C_{K g}^{(1)}\end{array}\right]$ where $\left\{\begin{array}{l}\mathcal{M} \equiv M_{N-1}^{N} M_{N-2}^{N-1} \cdots M_{1}^{2} \\ \text { See [12] for explicit } \\ \text { expression of } M_{p}^{p+1}\end{array}\right.$ Since the last layer goes to infinity, $C_{I e}^{(N)}=C_{I g}^{(N)}=0$. From the beam region, $C_{K g}^{(1)}=0$ and $C_{K e}^{(1)}=-i k Q Z_{0} I_{m}(k a / \gamma) /\left[\pi \beta \gamma^{2}\left(1+\delta_{m 0}\right)\right]$, one can easily solve for $C_{I e}^{(1)} \equiv-\alpha_{1} \frac{K_{m}^{(1)}}{I_{m}^{(1)}} C_{K e}^{(1)}=-C_{K e}^{(1)} \frac{\mathcal{M}_{12} \mathcal{M}_{33}-\mathcal{M}_{32} \mathcal{M}_{13}}{\mathcal{M}_{11} \mathcal{M}_{33}-\mathcal{M}_{13} \mathcal{M}_{31}}$, with $I_{m}^{(1)}=I_{m}\left(\nu^{(1)} b^{(1)}\right), I_{m}^{(1)}=I_{m}\left(\nu^{(1)} b^{(1)}\right)$. With beam at $r=a_{1}, \theta=0$, reduced forces on a unit test charge at $r=a_{2}>a_{1}$ and $\theta=\theta_{2}$ are $Z_{\|}=-\int d s E_{s}\left(a_{2}, \theta_{2}, s ; \omega\right) e^{-i k s}, \quad Z_{x}=-i \int d s\left[E_{x}\left(a_{2}, \theta_{2}, s ; \omega\right)-\beta G_{y}\left(a_{2}, \theta_{2}, s ; \omega\right)\right] e^{-i k s}$. Space-charge contributions for all multiples $\left(\alpha_{1}=1\right.$ or perfectly conducting at $\left.r=b^{(1)}\right)$ : $Z_{\|}^{\mathrm{SC}}=\sum_{m=0}^{\infty} \frac{i k Z_{0} L \cos m \theta_{2}}{\pi \beta \gamma^{2}\left(1+\delta_{m 0}\right)} I_{m}\left(x_{2}\right) \mathcal{K}_{m}\left(x_{1}\right), \quad \mathcal{K}_{m}\left(x_{i}\right)=\left[K_{m}\left(x_{i}\right)-\frac{K_{m}^{(1)}}{I_{m}^{(1)}} I_{m}\left(x_{i}\right)\right], \quad x_{i}=\frac{k a_{i}}{\gamma}$ $Z_{x}^{\mathrm{SC}}=\sum_{m=0}^{\infty} \frac{i k Z_{0} L}{\pi \beta \gamma^{3}\left(1+\delta_{m 0}\right)} I_{m}\left(x_{1}\right)\left[\cos \theta_{2} \cos m \theta_{2} \mathcal{K}_{m}\left(x_{2}\right)+\frac{m \gamma}{a_{2}} \sin \theta_{2} \sin m \theta_{2} \mathcal{K}_{m}^{\prime}\left(x_{2}\right)\right]$

The rest are from wall impedances. To any order $a_{1}^{n_{1}} a_{2}^{n_{2}}$, they are $Z_{\|, n_{1}, n_{2}}=-\frac{i L \mu_{0} \omega}{\pi \beta^{2} \gamma^{2}}\left(\frac{k a_{1}}{2 \gamma}\right)^{n_{1}}\left(\frac{k a_{2}}{2 \gamma}\right)^{n_{2}} \sum^{\prime} \frac{\cos m \theta_{2} \bar{\alpha}_{1}(m) K_{m}^{(1)} / I_{m}^{(1)}}{\left(1+\delta_{m 0}\right)\left(\frac{n_{1}-m}{2}\right) !\left(\frac{n_{1}+m}{2}\right) !\left(\frac{n_{2}-m}{2}\right) !\left(\frac{n_{2}+m}{2}\right) !}$
$Z_{x}^{W, n_{1}, n_{2}}=-\frac{i Z_{0} L}{\pi \beta \gamma^{2} a_{2}}\left(\frac{k a_{1}}{2 \gamma}\right)^{n_{1}}\left(\frac{k a_{2}}{2 \gamma}\right)^{n_{2}} \sum^{\prime} \frac{\left(n_{2} \cos \theta_{2} \cos m \theta_{2}+m \sin \theta_{2} \sin m \theta_{2}\right) \bar{\alpha}_{1}(m) K_{m}^{(1)} / I_{m}^{(1)}}{\left(1+\delta_{m 0}\right)\left(\frac{n_{1}-m}{2}\right) !\left(\frac{n_{1}+m}{2}\right) !\left(\frac{n_{2}-m}{2}\right) !\left(\frac{n_{2}+m}{2}\right) !}$ where $\bar{\alpha}_{1}(m) \equiv 1-\alpha_{1}(m), \sum^{\prime}$ implies from $m=0$ to $\min \left(n_{1}, n_{2}\right)$ with $n_{1}-n_{2}$ and $n_{1}-m$ even. The usual monopole and dipole pipe-wall impedances are $Z_{0}^{\|}=Z_{\|}^{W, 0,0}=\frac{i k Z_{0} L}{2 \pi \beta \gamma^{2}} \frac{\bar{\alpha}_{1} K_{0}^{(1)}}{I_{0}^{(1)}}$ and $Z_{1}^{\perp}=\frac{Z_{x}^{W, 1,1}}{a_{1}}=\frac{i L Z_{0} k^{2}}{4 \pi \beta \gamma^{4}} \frac{\bar{\alpha}_{1} K_{1}^{(1)}}{I_{1}^{(1)}}$

Multi-layer special cases: [13] Pipe wall: $b^{(1)}<r<b^{(2)}=b^{(1)}+t$.

Thin wall: Good for low frequencies. $t \rightarrow 0$ and $E_{s}$ does not change across wall. At $r=b^{(3)}$, Case PC: perfectly conducting, Case PM: perfectly magnetic, and Case INF: $b^{(2)} \rightarrow \infty$.

$$
\begin{aligned}
& \bar{\alpha}_{1}=-\frac{\gamma^{2} \beta^{2}\left(1-\alpha_{2}\right)+2 i x \gamma \beta / m \zeta}{1+\frac{x^{2}}{m^{2}}-\frac{i x \gamma \beta}{m}\left[\frac{2}{\zeta\left(1-\alpha_{2}\right)}+\frac{\zeta\left(1-\alpha_{2}\right)}{2}\right]}, \quad \alpha_{2}^{\mathrm{PC}}=-\alpha_{2}^{\mathrm{PM}}=\frac{K_{m}(y) I_{m}(x)}{K_{m}(x) I_{m}(y)} \underset{m \neq 0}{\approx}\left(\frac{b^{(1)}}{b^{(3)}}\right)^{2}, \quad \alpha_{2}^{\mathrm{INF}}=0 \\
& x=k b^{(1)} / \gamma, y=k b^{(3)} / \gamma, \zeta=Z_{0} \sigma_{c} t, \text { and } m \neq 0 .
\end{aligned}
$$


Thick wall: Good for high frequencies. At $r=b^{(2)}$, Case PC: perfectly conducting, Case PM: perfectly magnetic, and Case INF: $b^{(2)} \rightarrow \infty$. For $m \geq 1$,

$$
\begin{aligned}
& \bar{\alpha}_{1}=\frac{-2 \beta^{2} \gamma^{2}\left[1-\frac{(1+i) \Delta Q_{\eta}}{2 m \beta \gamma^{2}}\right]}{1-2 i p-\beta\left[\frac{(1-i) Q_{\alpha}}{m \Delta}-\frac{(1+i) \Delta Q_{\eta}}{2 m}\right]+\frac{Q_{\alpha} Q_{\eta}-m^{2} p^{2}}{m^{2} \gamma^{2}}}, \quad p=\frac{k^{2} \delta^{2}}{2}, \quad \Delta=\mu_{1} \beta k \delta_{c}, \\
& Q_{\alpha}=k b^{(1)} \frac{Q_{2}-\alpha_{2} P_{2}}{1-\alpha_{2}}, \quad Q_{\eta}=k b^{(1)} \frac{Q_{2}-\eta_{2} P_{2}}{1-\eta_{2}}, \quad Q_{2}=\frac{K_{m}^{\prime(2)}}{K_{m}^{(2)}}, \quad P_{2}=\frac{I_{m}^{\prime(2)}}{I_{m}^{(2)}}
\end{aligned}
$$

Boundary conditions require $\alpha_{2}^{\mathrm{PC}}=\eta_{2}^{\mathrm{PM}}=\frac{K_{m}^{(2,3)} I_{m}^{(2)}}{I_{m}^{(2,3)} K_{m}^{(2)}}, \quad \eta_{2}^{\mathrm{PC}}=\alpha_{2}^{\mathrm{PM}}=\frac{K_{m}^{\prime(2,3)} I_{m}^{(2)}}{I_{m}^{\prime(2,3)} K_{m}^{(2)}}$,

$\alpha_{2}^{\mathrm{INF}}=\eta_{2}^{\mathrm{INF}}=0$, with $I_{m}^{(p+1, p)}=I_{m}\left(\nu^{(p+1)} b^{(p)}\right), I_{m}^{(p)}=I_{m}\left(\nu^{(p)} b^{(p)}\right)$ and similar definitions for $I_{m}^{\prime}, K_{m}$, and $K_{m}^{\prime}$.

Electric- and magnetic-dipole approximation: $\bar{\alpha}_{1}$ can also be derived [14] by approxi-

\begin{tabular}{|c|c|c|}
\hline Desc & Impedances & Wakes \\
\hline \multicolumn{3}{|c|}{$\begin{array}{l}\text { High frequency optical model: [15] High frequency } k \gg 1 / h \text {, short-range }-z \ll h \text {, } \\
\text { transition length } L \ll k h^{2}, h \text { is minimum aperture. For tapered transition of angle } \theta \text {, need } \\
k \gg 1 / h \theta \text {. }\end{array}$} \\
\hline \multirow{3}{*}{$\begin{array}{l}\text { Transitions, shallow } \\
\text { cavities, collimators, } \\
\text { irises: } \\
\text { (a) Axially symmet- } \\
\text { ric examples: }[16]- \\
{[18] \text { (i) step-in tran- }} \\
\text { sition (from } d \text { to } b \text { ), } \\
\text { (ii) step-out (from } b \\
\text { to } d \text { ), long collima- } \\
\text { tor, shallow cavity } \\
\text { with gap g, (iii) thin } \\
\text { iris } \\
\text { (b) } 3 \mathrm{D}, \text { mirror sym- } \\
\text { metric in } x, y \text { : }[15] \\
\text { (i) flat step-out tran- } \\
\text { sition, aperture } 2 b \\
\text { to } 2 d, \text { (ii) any step- } \\
\text { in transition; iris } \\
\text { with small (iii) flat } \\
\text { (height } 2 b \text { ), (iv) el- } \\
\text { liptical (axes } w \text { by } b \text { ), } \\
\text { aperture }\end{array}$} & $\begin{array}{l}Z^{\|} \text {and } k Z^{\perp} \text { are both constants } \\
\text { similar for } k Z_{d}^{\perp}, k Z_{q}^{\perp}, W_{d}^{\perp}, W_{q}^{\perp}\end{array}$ & $\begin{array}{c}W_{\|}=-Z^{\|} c \delta(z) \\
W_{\perp}=-k Z^{\perp} c H(-z)\end{array}$ \\
\hline & $\begin{array}{l}\text { (i) } Z_{0}^{\|}=Z_{1}^{\perp}=0, \quad \text { (ii) } Z_{0}^{\|}= \\
\text {(iii) } Z_{0}^{\|}=\frac{Z_{0}}{\pi} \ln \frac{d}{b}, \\
\text { where } b \text { is small iris or pipe radiu } \\
\text { shallow cavity, waves reflect from } \\
\text { collimator, bottom length } \gg k b^{2}\end{array}$ & $\begin{array}{l}\frac{d}{b}, \quad k Z_{1}^{\perp}=\frac{Z_{0}}{\pi}\left(\frac{1}{b^{2}}-\frac{1}{d^{2}}\right) \\
\frac{\perp}{1}=\frac{Z_{0}}{2 \pi}\left(\frac{1}{b^{2}}-\frac{b^{2}}{d^{4}}\right) \\
\text { is large pipe radius. Note: for } \\
\text { iter wall } \Rightarrow g \gtrsim k(d-b)^{2} ; \text { for }\end{array}$ \\
\hline & $\begin{array}{r}\text { (i) } k Z_{y}=\frac{\pi}{8} Z_{0}\left(\frac{1}{b^{2}}-\frac{1}{d}\right. \\
\text { (ii) } Z_{\|}=Z_{\perp}=0, \quad \text { (iii) } k 2 \\
\text { (iv) } k Z_{y}=\frac{Z_{0}}{2 \pi b^{2}}, \quad k Z_{d}^{y}=\frac{Z_{0}}{4 \pi b^{2}}\end{array}$ & $\begin{array}{l}, \quad Z_{q}^{y}=\frac{1}{2} Z_{d}^{y}=\frac{1}{3} Z_{y} \\
=\frac{Z_{0}}{2 \pi b^{2}}, \quad Z_{q}^{y}=Z_{d}^{y}=\frac{1}{2} Z_{y} \\
\left.-\frac{b^{2}}{w^{2}}\right), \quad k Z_{q}^{y}=\frac{Z_{0}}{4 \pi b^{2}}\left(1-\frac{b^{2}}{w^{2}}\right)\end{array}$ \\
\hline $\begin{array}{l}\text { High frequency } \\
\text { diffraction formu- } \\
\text { lae: } k \gg 1 / b \\
\text { (a) Deep cavity (Fres- } \\
\text { nel diffraction) } \\
{[19,1] \text {, cavity radius }} \\
d \text { and gap } g \text {. }\end{array}$ & $\begin{array}{l}\quad Z_{m}^{\|}=\frac{\sqrt{2} Z_{0}}{\left(1+\delta_{m 0}\right) \pi^{3 / 2} b^{2 m+1}} \sqrt{\frac{i g}{k}} \\
\qquad Z_{1}^{\perp}=\frac{2}{b^{2} k} Z_{0}^{\|} \\
\text {Note: no reflections from } \\
\text { outer wall } \Rightarrow g \lesssim k(d-b)^{2} .\end{array}$ & $\begin{array}{c}W_{m}^{\prime}=\frac{\sqrt{2} Z_{0} c}{\left(1+\delta_{m 0}\right) \pi^{2} b^{2 m+1}} \sqrt{\frac{g}{-z}} \\
W_{1}=\frac{2}{b^{2}} \int W_{0}^{\prime} d z\end{array}$ \\
\hline
\end{tabular}
mating beam dipole motion as a superposition of oscillating electric and magnetic dipoles. 


\begin{tabular}{|c|c|}
\hline Description & Impedances \\
\hline $\begin{array}{l}\text { (b) Periodic array of } \\
\text { deep cavities (model } \\
\text { for linear accelera- } \\
\text { tor structures): }[20]- \\
\text { [24] period } L \text {, gap } g \text {, } \\
\text { outer cavity radius } d \text {, } \\
\text { with } g \lesssim k(d-b)^{2} \text {. }\end{array}$ & $\begin{array}{c}\frac{Z_{0}^{\|}}{L}=\frac{i Z_{0}}{\pi k b^{2}}\left[1+\frac{\alpha(g / L) L}{b} \sqrt{\frac{2 \pi i}{k g}}\right]^{-1} \\
\alpha(\zeta) \approx 1-0.465 \sqrt{\zeta}-0.070 \zeta \\
Z_{1}^{\perp}=\frac{2}{b^{2} k} Z_{0}^{\|}\end{array}$ \\
\hline $\begin{array}{l}\text { Numerical fit: }[24,25] \\
\text { valid over larger } z \\
\text { range: }-z / L \leq 0.15 \\
0.34 \leq b / L \leq 0.69 \\
0.54 \leq g / L \leq 0.89\end{array}$ & $\begin{array}{c}W_{0}^{\prime}=\frac{Z_{0} c}{\pi b^{2}} \exp \left(-\sqrt{\frac{z}{z_{0}}}\right), W_{1}=\frac{4 Z_{0} c z_{1}}{\pi b^{4}}\left[1-\left(1+\sqrt{\frac{z}{z_{1}}}\right) \exp \left(-\sqrt{\frac{z}{z_{1}}}\right)\right] \\
z_{0}=-0.41 \frac{b^{1.8} g^{1.6}}{L^{2.4}}, \quad z_{1}=-0.17 \frac{b^{1.79} g^{0.38}}{L^{1.17}}\end{array}$ \\
\hline $\begin{array}{l}\text { Bethe's dipole } \\
\text { moments of a hole } \\
\text { of radius } a \text { on beam } \\
\text { pipe wall }[26] .\end{array}$ & $\begin{array}{l}\text { Electric and magnetic dipole } \quad \vec{d}=-\frac{2 \epsilon_{0}}{3} a^{3} \vec{E}, \vec{m}=-\frac{4}{3 \mu_{0}} a^{3} \vec{B} \\
\text { moments when wavelength } \gg a: \\
\vec{E} \text { and } \vec{B} \text { are electric and magnetic flux density at hole when hole } \\
\text { is absent. This is a diffraction solution for a thin-wall pipe. }\end{array}$ \\
\hline $\begin{array}{l}\text { Small 3D obstacle } \\
\text { on beam pipe: }[27, \\
28] \text { size } \ll b \text {, low }\end{array}$ & $\begin{array}{c}W_{0}^{\prime}=-c^{2} \mathcal{L} \delta^{\prime}(z) \\
W_{1}=\frac{4}{b^{2}} \int W_{0}^{\prime} \cos \phi d z\end{array}$ \\
\hline $\begin{array}{l}\phi \text { azimuth } \\
\text { position of }\end{array}$ & $\begin{array}{c}\text { Inductance } \mathcal{L}=\frac{Z_{0}\left(\alpha_{e}+\alpha_{m}\right)}{4 \pi^{2} b^{2} c} \\
\alpha_{e} \text { is electric polarizability, } \alpha_{m} \text { magnetic susceptibility }\end{array}$ \\
\hline $\begin{array}{l}\text { Elliptical hole: ma- } \\
\text { jor and minor radii } \\
\text { are } a \text { and } d . K(m) \\
\text { and } E(m) \text { are com- } \\
\text { plete elliptical func- } \\
\text { tions of the first and } \\
\text { second kind, with } \\
m=1-m_{1} \text { and } \\
m_{1}=(d / a)^{2} . \text { For } \\
\text { long ellipse perpen- } \\
\text { dicular to beam, ma- } \\
\text { jor axis } a \ll b, \text { beam } \\
\text { pipe radius, because } \\
\text { the curvature of the } \\
\text { beam pipe has been } \\
\text { neglected here }[29]\end{array}$ & 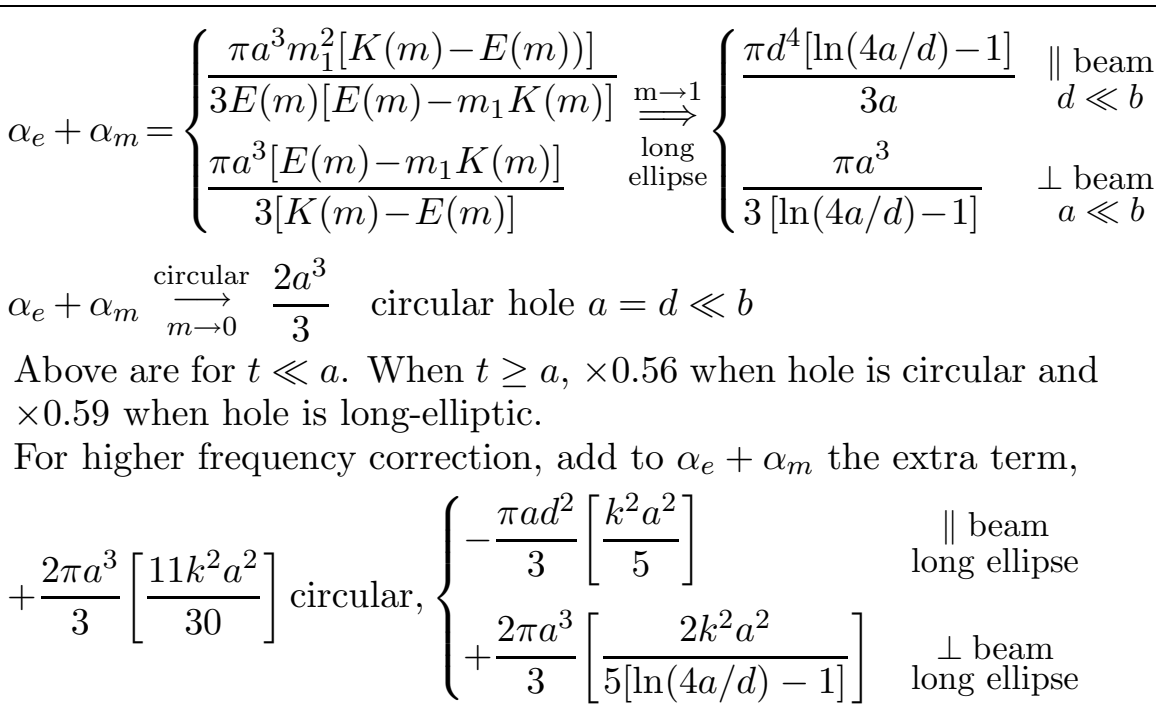 \\
\hline & $\alpha_{e}+\alpha_{m}=w^{3}(0.1814-0.0344 w / L) \quad t \ll a, \quad \times 0.59$ when $t \geq a$ \\
\hline & $\alpha_{e}+\alpha_{m}=w^{3}(0.1334-0.0500 w / L)$ \\
\hline
\end{tabular}




\begin{tabular}{|c|c|}
\hline Description & Impedances \\
\hline $\begin{array}{l}\text { Annular-ring-shaped } \\
\text { cut: inner and outer } \\
\text { radii } a \text { and } d=a+w \\
\text { with } w \ll d \text {. }\end{array}$ & $\begin{array}{c}\alpha_{e}+\alpha_{m}=\frac{\pi^{2} d^{2} a}{2 \ln (32 d / w)-4}-\frac{\pi^{2} w^{2}(a+d)}{16} \quad t \ll d \\
\alpha_{e}+\alpha_{m}=\pi d^{2} w-\frac{1}{2} w^{2}(a+d) \quad t \geq d\end{array}$ \\
\hline $\begin{array}{l}\text { Half ellipsoidal pro- } \\
\text { trusion with semi } \\
\text { axes } h \text { radially, } a \\
\text { longitudinally, and } d \\
\text { azimuthally. }{ }_{2} F_{1} \text { is } \\
\text { the hypergeometric } \\
\text { function. }\end{array}$ & $\begin{array}{c}\alpha_{e}+\alpha_{m}=2 \pi a h d\left[\frac{1}{I_{b}}+\frac{1}{I_{c}-3}\right] \\
I_{b}={ }_{2} F_{1}\left(1,1 ; \frac{5}{2} ; 1-\frac{h^{2}}{a^{2}}\right), \quad I_{c}={ }_{2} F_{1}\left(1, \frac{1}{2} ; \frac{5}{2} ; 1-\frac{a^{2}}{h^{2}}\right), \quad \text { if } a=d \\
\alpha_{e}+\alpha_{m}=\pi a^{3} \quad \text { if } a=d=h, \quad \frac{2 \pi h^{3}}{3[\ln (2 h / a)-1]} \quad \text { if } a=d \ll h \\
\alpha_{e}+\alpha_{m}=\frac{8 h^{3}}{3}\left[1+\left(\frac{4}{\pi}-\frac{\pi}{4}\right) \frac{a}{h}\right] \quad \text { if } a \ll h=d \\
\alpha_{e}+\alpha_{m}=\frac{8 \pi h^{4}}{3 a}\left[\ln \frac{2 a}{h}-1\right] \quad \text { if } a \gg h=d\end{array}$ \\
\hline $\begin{array}{l}\text { Small inductive } \\
\text { objects-2D: }[27,30]\end{array}$ & $\begin{array}{l}Z_{0}^{\|}=-i k c \mathcal{L}, \quad Z_{1}^{\perp}=\frac{2}{b^{2} k} Z_{0}^{\|} \quad W_{0}^{\prime}=-c^{2} \mathcal{L} \delta^{\prime}(z), W_{1}=\frac{2}{b^{2}} \int W_{0}^{\prime} d z\end{array}$ \\
\hline $\begin{array}{l}\text { low irises, and transi- } \\
\text { tions at low freq. } \\
(h \ll b, k \ll 1 / h) ; h \\
\text { is height of object, } \\
g \text { is gap of cavity } \\
\text { or length of iris; } \mathcal{L} \\
\text { is inductance. For } \\
\text { tapered transition } \\
\text { pair: } \theta \text { is taper angle. }\end{array}$ & $\begin{array}{c}\text { Pill box } g \lesssim h \ll b: \mathcal{L}=\frac{Z_{0}}{2 \pi c b}\left[g h-\frac{g^{2}}{2 \pi}\right] \\
\text { Shallow iris } g \lesssim h \ll b: \mathcal{L}=\frac{Z_{0} h^{2}}{4 c b} \\
\text { Transition pair } g \gg h, h \ll b: \mathcal{L}=\frac{Z_{0} h^{2}}{2 \pi^{2} c b}\left(\ln \frac{2 \pi b}{h}+\frac{1}{2}\right) \\
\text { Tapered }: \mathcal{L}=\frac{Z_{0} h^{2}}{\pi^{2} c b}\left[\ln \left(\frac{b \theta}{h}-2 \theta \cot \theta\right)+\frac{3}{2}-\gamma_{e}-\psi\left(\frac{\theta}{\pi}\right)-\frac{\pi}{2} \cot \theta-\frac{\pi}{2 \theta}\right] \\
\gamma_{e} \approx 0.57721 \text { is Euler's constant, } \psi(x) \text { is psi function. }\end{array}$ \\
\hline $\begin{array}{l}\text { Wall roughness } \\
\text { inductive model: } \\
{[35] 1-\mathrm{D} \text { axisymmet- }} \\
\text { ric bump on beam } \\
\text { pipe, } h(z) \text { or } 2 \text {-D } \\
\text { bump } h(z, \theta) \text {. Valid } \\
\text { for low frequency } \\
k \ll(\text { bump length } \\
\text { or width })^{-1}, h \ll b \text {, } \\
\text { and }|\nabla h| \ll 1 \text {. See } \\
\text { also }[36]\end{array}$ & $\begin{array}{l}\text { 1-D: } \quad Z_{0}^{\|}=-\frac{2 i k Z_{0}}{b} \int_{0}^{\infty} \kappa|\tilde{h}(\kappa)|^{2} d \kappa \\
\text { with spectrum } \quad \tilde{h}(k)=\frac{1}{2 \pi} \int_{-\infty}^{\infty} h(z) e^{-i k z} d z \\
\text { 2-D: } \quad Z_{0}^{\|}=-\frac{4 i k Z_{0}}{b} \sum_{m=-\infty}^{\infty} \int_{-\infty}^{\infty} \frac{\kappa^{2}}{\sqrt{\kappa^{2}+m^{2} / b^{2}}}\left|\tilde{h}_{m}(\kappa)\right|^{2} d \kappa \\
\text { with spectrum } \tilde{h}_{m}(k)=\frac{1}{(2 \pi)^{2}} \int_{0}^{2 \pi} d \theta \int_{-\infty}^{\infty} d z h(z, \theta) e^{-i k z-i m \theta} \\
\text { Note: small periodic corrugations model is also used for wall rough- } \\
\text { ness impedance estimation. }\end{array}$ \\
\hline $\begin{array}{l}\text { Small periodic } \\
\text { corrugations: } \quad(\mathrm{a}) \\
{[31,32] L \lesssim h \ll b,} \\
k \ll 1 / h ; L \text { period, } \\
h \text { depth, g gap, } \wp \\
\text { principal value; } \beta_{g} c \\
\text { group velocity. }\end{array}$ & $\begin{array}{ll}\frac{Z_{0}^{\|}}{L}=\frac{Z_{0}}{\pi b^{2}}\left[\pi k_{r} \delta\left(k^{2}-k_{r}^{2}\right)+i \wp\left(\frac{k}{k^{2}-k_{r}^{2}}\right)\right], \quad \frac{W_{0}^{\prime}}{L}=\frac{Z_{0} c}{\pi b^{2}} \cos k_{r} z \\
Z_{1}^{\perp}=\frac{2}{b^{2} k} Z_{0}^{\|}, \quad k_{r}=\sqrt{\frac{2 L}{b g h}} ;\left(1-\beta_{g}\right)=\frac{4 h g}{b L}, \quad W_{1}=\frac{2}{b^{2}} \int W_{0}^{\prime} d z\end{array}$ \\
\hline $\begin{array}{l}\text { (b) }[33] L \gg h, L \ll b \\
k \ll 1 / h ; k_{L}=2 \pi / L\end{array}$ & $\frac{W_{0}^{\prime}}{L}=-\frac{Z_{0} c h^{2} k_{L}^{3}}{16 \pi^{3 / 2} b} \frac{1}{\left(-k_{L} z\right)^{3 / 2}}$ \\
\hline
\end{tabular}




\begin{tabular}{|c|c|c|}
\hline Description & Impedances & Wakes \\
\hline $\begin{array}{l}\text { Thin dielectric or } \\
\text { ferrite layer on } \\
\text { pipe: }[34] \text { thickness } \\
h \ll b \text {. }\end{array}$ & \multicolumn{2}{|c|}{$\begin{array}{l}\text { Like small periodic corrugations }(\mathrm{a}) \text {, but } k_{r}=\left[\frac{2 \epsilon_{r}}{\left(\epsilon_{r} \mu_{r}-1\right) b h}\right]^{1 / 2}, \\
\text { with relative dielectric constant } \epsilon_{r} \text { and magnetic permeability } \mu_{r} \text {. }\end{array}$} \\
\hline \multirow{2}{*}{$\begin{array}{l}\text { Coherent syn- } \\
\text { chrotron radiation } \\
\text { (CSR): [37]-[39] } \\
\text { Bunch moves in free } \\
\text { space on a circle of } \\
\text { radius } R ; k \ll \gamma^{3} / R \text {. } \\
\text { See Sec.??. }\end{array}$} & $\frac{Z_{0}^{\|}}{L}=\frac{Z_{0}}{2 \cdot 3^{1 / 3} \pi} \Gamma\left(\frac{2}{3}\right)\left[\frac{i k}{R^{2}}\right]^{1 / 3}$ & $\frac{W_{0}^{\prime}}{L}=-\frac{Z_{0} c}{2 \cdot 3^{4 / 3} \pi R^{2 / 3}} \frac{1}{z^{4 / 3}}$ \\
\hline & \multicolumn{2}{|c|}{$\begin{array}{l}\Gamma(2 / 3) \approx 1.3541 . \text { Note: non-zero wake for test particle ahead of } \\
\text { driving particle. } W_{0}^{\prime}\left(0^{+}\right) / L \approx 0.1 Z_{0} c \gamma^{4} / R^{2} \text {. This is also used } \\
\text { to approximate effect at high } k \text { for beam in beam pipe; shielded } \\
\text { (suppressed) for } k \lesssim R^{1 / 2} b^{-3 / 2} \text {. }\end{array}$} \\
\hline $\begin{array}{l}\text { Round collimator: } \\
\text { (a) }[40] \text { low frequen- } \\
\text { cy } k \ll 1 / d \text {. }\end{array}$ & $\begin{array}{l}Z_{1}^{\perp}=-0.3 i \frac{Z_{0}}{d} \\
\text { collimator radius } d \ll b\end{array}$ & $\begin{array}{l}W_{1}=-0.3 \frac{Z_{0} c}{d} \delta(z) \\
\text { collimator radius } d \ll b\end{array}$ \\
\hline $\begin{array}{l}\text { (b) High frequency } \\
k \gg 1 / d ; \text { if tapered, } \\
\text { angle } \theta \gg 1 /(k d)\end{array}$ & \multicolumn{2}{|l|}{ See optical model formulae (a) above } \\
\hline $\begin{array}{l}\text { (c) [41] For any fre- } \\
\text { quency, small angle, } \\
d^{\prime}(s) \ll 1, k d d^{\prime} \ll 1 \text {, } \\
\text { with } d(s) \text { pipe pro- } \\
\text { file versus longitudi- } \\
\text { nal position } s, \text { and } \\
d^{\prime} \text { is derivative of } d \\
\text { with respect to } s \text {. }\end{array}$ & $\begin{array}{c}Z_{0}^{\|}=\frac{-i Z_{0} k}{4 \pi} \int d s\left(d^{\prime}\right)^{2} \\
Z_{1}^{\perp}=\frac{-i Z_{0}}{2 \pi} \int d s\left(\frac{d^{\prime}}{d}\right)^{2} \\
\Rightarrow \text { symm. tapers of angle } \theta \ll 1: \\
Z_{1}^{\perp}=\frac{-i Z_{0}}{\pi} \theta\left(\frac{1}{d}-\frac{1}{b}\right)\end{array}$ & $\begin{array}{c}W_{0}^{\prime}=\frac{Z_{0} c}{4 \pi} \int d s\left(d^{\prime}\right)^{2} \delta^{\prime}(z) \\
W_{1}=-\frac{Z_{0} c}{2 \pi} \int d s\left(\frac{d^{\prime}}{d}\right)^{2} \delta(z) \\
W_{1}=-\frac{Z_{0} c}{\pi} \theta\left(\frac{1}{d}-\frac{1}{b}\right) \delta(z)\end{array}$ \\
\hline $\begin{array}{l}\text { Flat collimator: }[42] \\
\text { low frequency, small } \\
\text { angle, } h^{\prime}(s) \ll 1, \\
h \ll w \ll \ell \text {, with } h(s) \\
\text { vertical profile, } w \\
\text { width, } \ell \text { length }\end{array}$ & $Z_{y}=\frac{-i Z_{0} w}{4} \int d s \frac{\left(h^{\prime}\right)^{2}}{h^{3}}$ & $W_{y}=-\frac{Z_{0} c w}{4} \int d s \frac{\left(h^{\prime}\right)^{2}}{h^{3}} \delta(z)$ \\
\hline \multirow{2}{*}{$\begin{array}{l}\text { Pill-box cavity }- \\
\text { low frequency: }[43] \\
\text { cavity radius } d, \text { gap } \\
g ; S=d / b \text {. When } \\
g \gg 2(d-b) \text {, replace } \\
g \text { by } d-b \text {. Valid for } \\
k \ll 1 / d \text {. }\end{array}$} & $\begin{aligned} Z_{0}^{\|} & =-i k \frac{Z_{0} g}{2 \pi} \ln S \\
Z_{1}^{\perp} & =-i \frac{Z_{0} g}{\pi b^{2}} \frac{S^{2}-1}{S^{2}+1}\end{aligned}$ & $\begin{array}{c}W_{0}^{\prime}=-\frac{Z_{0} c g}{2 \pi} \ln S \delta^{\prime}(z) \\
W_{1}=-\frac{Z_{0} c g}{\pi b^{2}} \frac{S^{2}-1}{S^{2}+1} \delta(z)\end{array}$ \\
\hline & \multicolumn{2}{|c|}{$\begin{array}{l}\text { Effect will be one half for a step in the beam pipe from radius } b \\
\text { to radius } d \text {, or vice versa, when } g \gg 2(d-b) \text {. }\end{array}$} \\
\hline \multirow[t]{2}{*}{$\begin{array}{l}\text { Resonator model: } \\
\text { [1] for } m \text {-th azimuth- } \\
\text { al mode, with shunt } \\
\text { impedance } R_{s}^{(m)} \text {, } \\
\text { quality factor } Q \text {, and } \\
\text { resonant frequency } \\
k_{r} \text {. }\end{array}$} & $\begin{aligned} Z_{m}^{\|} & =\frac{R_{s}^{(m)}}{1+i Q\left(k_{r} / k-k / k_{r}\right)} \\
Z_{m}^{\perp} & =\frac{R_{s}^{(m)} / k}{1+i Q\left(k_{r} / k-k / k_{r}\right)}\end{aligned}$ & $\begin{aligned} W_{m} & =\frac{R_{s}^{(m)} c k_{r}}{Q \bar{k}_{r}} e^{\alpha z} \sin \bar{k}_{r} z \\
\text { where } \alpha & =k_{r} /(2 Q) \\
\bar{k}_{r} & =\sqrt{\left|k_{r}^{2}-\alpha^{2}\right|}\end{aligned}$ \\
\hline & \multicolumn{2}{|c|}{$\begin{array}{l}\text { Valid only close to } k_{r} \text {. As } k \rightarrow \infty, Z_{0}^{\|} \rightarrow k^{-1 / 2} \text { for non-periodic } \\
\text { cavities and } \rightarrow k^{-3 / 2} \text { for an infinite array of cavities. }[16,46]\end{array}$} \\
\hline
\end{tabular}




\begin{tabular}{|c|c|c|}
\hline tion & Impedances & Wa \\
\hline $\begin{array}{l}\text { Closed pill-box } \\
\text { cavity: [44] res- } \\
\text { onant frequencies } \\
k_{m n p} \text { and "circuit" } \\
\left(R_{s} / Q\right)_{m n p}[45], \\
\text { where } m, n, p \text {, are } \\
\text { azimuthal, radial, } \\
\text { longitudinal mode } \\
\text { numbers. Cavity } \\
\text { radius } d \text { and length } \\
g ; x_{m n} \text { is } n^{\text {th }} \text { zero of } \\
\text { Bessel function } J_{m} .\end{array}$ & $\begin{array}{l}{\left[\frac{R_{s}}{Q}\right]_{0 n p}=\frac{Z_{0}}{x_{0 n}^{2} J_{0}^{\prime 2}\left(x_{0 n}\right)} \frac{8}{\pi g k_{0 n p}}} \\
{\left[\frac{R_{s}}{Q}\right]_{1 n p}=\frac{Z_{0}}{J_{1}^{\prime 2}\left(x_{1 n}\right)} \frac{2}{\pi g d^{2} k_{1 n p}^{2}}}\end{array}$ & $\begin{array}{l}+\frac{p^{2} \pi^{2}}{g^{2}} \\
\sin ^{2} \frac{g k_{0 n p}}{2 \beta} \times \frac{1}{1+\delta_{0 p}} \quad p \text { even } \\
\cos ^{2} \frac{g k_{0 n p}}{2 \beta} \\
\sin ^{2} \frac{g k_{1 n p}}{2 \beta} \quad p \neq 0 \text { odd } \\
\cos ^{2} \frac{g k_{1 n p}}{2 \beta} \quad p \text { odd }\end{array}$ \\
\hline $\begin{array}{l}\text { Curvature impe- } \\
\text { dance: [47] Smooth } \\
\text { toroidal chamber } \\
\text { of rectangular cross } \\
\text { section, width } b-a \text {, } \\
\text { height } h, \text { inner ra- } \\
\text { dius } a, \text { outer radius } \\
b, \text { and } R=\frac{1}{2}(a+b) \text {. } \\
\text { As Lorentz factor } \\
\gamma \rightarrow \infty, \text { a contribu- } \\
\text { tion remains. }\end{array}$ & 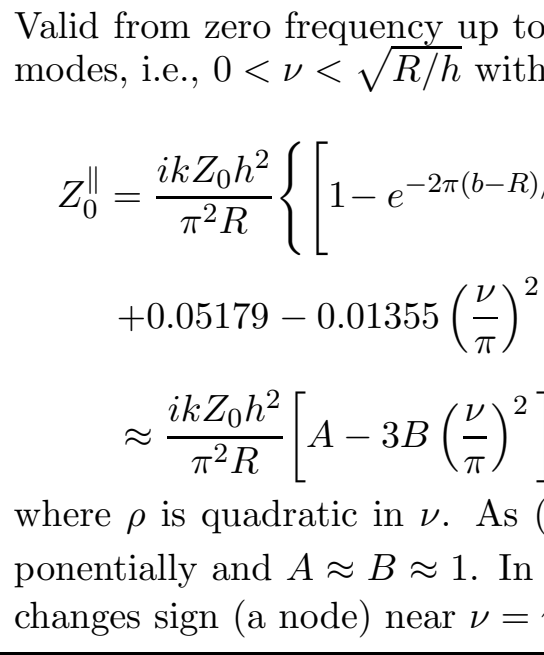 & $\begin{array}{l}\text { ist below synchronous resonant } \\
=k h, \\
\left.-e^{-2 \pi(R-a) / h}\right]\left[1-3\left(\frac{\nu}{\pi}\right)^{2}\right] \\
+\rho k R \\
-a) / h \text { increases, } \rho \text { vanishes ex- } \\
\text { neral, } A / B \approx 1 \text { implying } \operatorname{Im} Z_{0}^{\|} \\
\sqrt{3} .\end{array}$ \\
\hline \multirow{2}{*}{$\begin{array}{l}\text { Kicker with window- } \\
\text { frame magnet: }[49] \\
\text { width } a, \text { height } b, \\
\text { length } L \text {, beam offset } \\
x_{0} \text { horizontally, and } \\
\text { all image current } \\
\text { carried by conduct- } \\
\text { ing current plates. }\end{array}$} & $\begin{array}{c}Z_{0}^{\|}=\frac{k^{2} c^{2} \mu_{0}^{2} L^{2} x_{0}^{2}}{4 a^{2} Z_{k}} \\
Z_{1}^{\perp}=\frac{k c^{2} \mu_{0}^{2} L^{2}}{4 a^{2} Z_{k}}\end{array}$ & $\begin{array}{c}W_{0}^{\prime}=-\frac{c^{3} \mu_{0}^{2} L^{2} x_{0}^{2}}{4 a^{2} Z_{k}} \delta_{0}^{\prime \prime}(z) \\
W_{1}=-\frac{c^{3} \mu_{0}^{2} L^{2}}{4 a^{2} Z_{k}} \delta^{\prime}(z)\end{array}$ \\
\hline & \multicolumn{2}{|c|}{$\begin{array}{l}Z_{k}=-i k c \mathcal{L}+Z_{g} \text { with } \mathcal{L} \approx \mu_{0} b L / a \text { the inductance of the windings } \\
\text { and } Z_{g} \text { the impedance of the generator and the cable. If the kicker } \\
\text { is of C-type magnet, } x_{0} \text { in } Z_{0}^{\|} \text {should be replaced by }\left(x_{0}+b\right) .\end{array}$} \\
\hline \multirow{2}{*}{$\begin{array}{l}\text { Traveling-wave } \\
\text { kicker }[49] \text { with } \\
\text { characteristic impe- } \\
\text { dance } Z_{c} \text { for the } \\
\text { cable, and a window } \\
\text { magnet of width } a \text {, } \\
\text { height } b \text {, and length } \\
L \text {. Valid for fre- } \\
\text { quency below cutoff. }\end{array}$} & $\begin{array}{c}Z_{0}^{\|}=\frac{Z_{c}}{4}\left[2 \sin ^{2} \frac{\theta}{2}-i \sin \theta\right] \\
Z_{1}^{\perp}=\frac{Z_{c} L}{4 a b}\left[\frac{1-\cos \theta}{\theta}-i \frac{\sin \theta}{\theta}\right]\end{array}$ & $\begin{array}{c}W_{0}^{\prime}=\frac{Z_{c} c}{4}\left[\delta(z)-\delta\left(z+\frac{L}{\beta_{\mathrm{ph}}}\right)\right] \\
W_{1}=\frac{Z_{c} \beta c}{4 a b}\left[H(z)-H\left(z+\frac{L}{\beta_{\mathrm{ph}}}\right)\right]\end{array}$ \\
\hline & \multicolumn{2}{|c|}{$\begin{array}{l}\theta=k L / \beta_{\mathrm{ph}} \text { denotes the electrical length of the kicker windings and } \\
\beta_{\mathrm{ph}} c=Z_{c} a c /\left(Z_{0} b\right) \text { is the matched transmission-line phase velocity } \\
\text { of the capacitance-loaded windings. Here, } \beta_{\mathrm{ph}} \ll \beta \rightarrow 1 \text {, the beam } \\
\text { velocity. }\end{array}$} \\
\hline
\end{tabular}




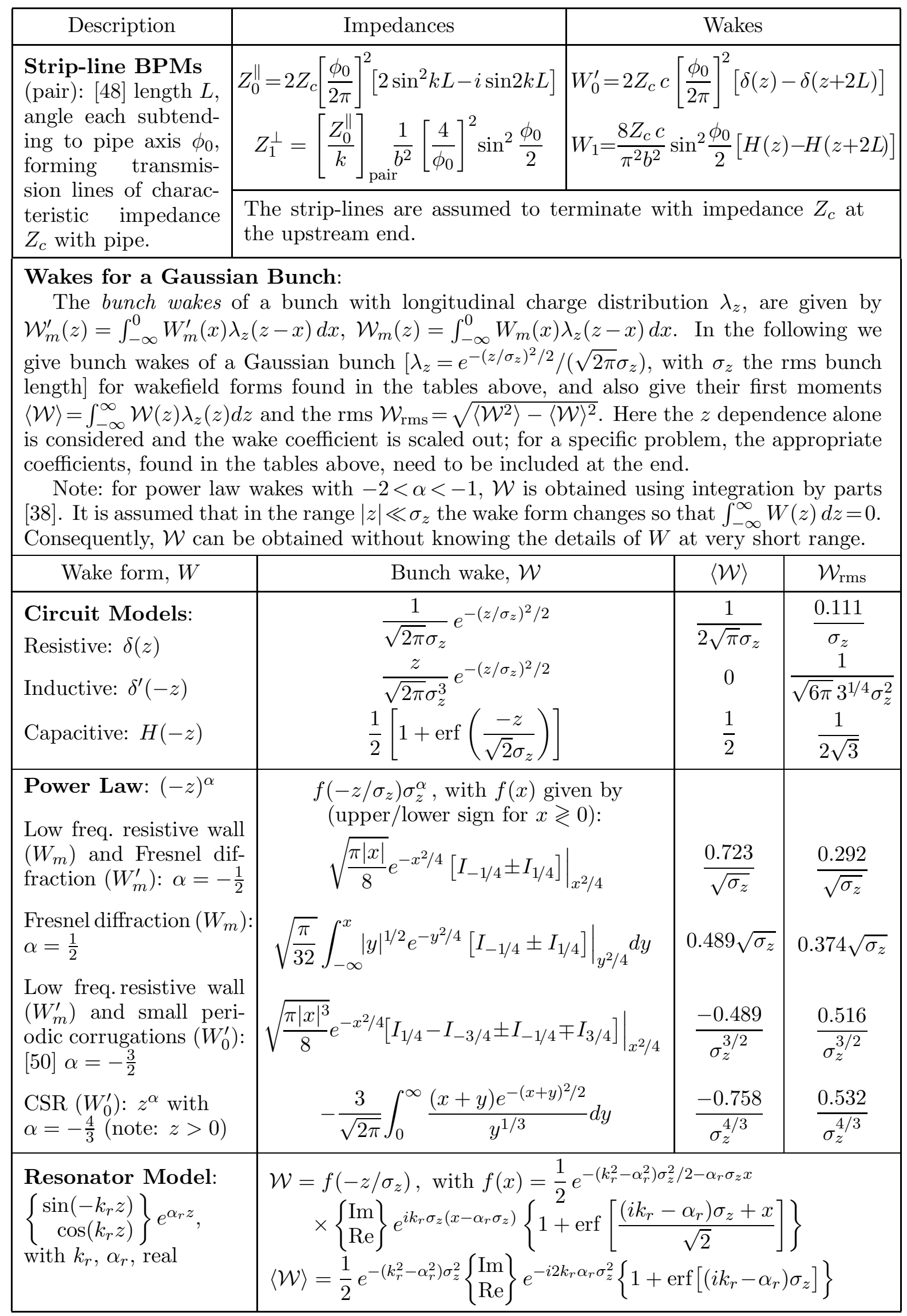

